\title{
Small fibre neuropathy in patients with chronic pain and a previous diagnosis of Multiple Chemical Sensitivity syndrome
}

Enrico Fileccia ( $\nabla$ enricofileccia@gmail.com )

IRCCS Istituto delle Scienze Neurologiche di Bologna, UOC Clinica Neurologica

Alex Incensi

IRCCS Istituto delle Scienze Neurologiche di Bologna, UOC Clinica Neurologica

Francesco Ventruto

Università di Bologna

Giovanni Rizzo

IRCCS Istituto delle Scienze Neurologiche di Bologna, UOC Clinica Neurologica

\section{Damiano Galimberti}

Università di Catania, Dipartimento di Biochimica e Patologia Clinica

\section{Giacomo Rao}

Sovrintendenza Sanitaria Centrale Settore Prevenzione Ricerca Direzione Generale INAIL Roma

\section{Fabrizio Salvi}

IRCCS Istituto delle Scienze Neurologiche di Bologna, UOC Clinica Neurologica

\section{Rocco Liguori}

Università di Bologna

\section{Vincenzo Donadio}

IRCCS Istituto delle Scienze Neurologiche di Bologna, UOC Clinica Neurologica

\section{Research Article}

Keywords: SFN, Multiple chemical sensitivity syndrome (MCS) , sensory, mainly pain, autonomic symptoms

Posted Date: May 6th, 2021

DOI: https://doi.org/10.21203/rs.3.rs-479848/v1

License: (1) (1) This work is licensed under a Creative Commons Attribution 4.0 International License. Read Full License 


\section{Abstract}

Small fiber neuropathy (SFN) is characterised by an involvement of A $\delta$ and C-fibres leading to sensory, mainly pain, and/or autonomic symptoms. Multiple chemical sensitivity syndrome (MCS) is a still not fully defined condition characterised by the arising of different symptoms after exposure to several chemical substances. Pain is often complained by patients affected by MCS. In this study we report the histological and clinical data of a cohort of patients referred to our attention for the suspect of SFN because of chronic pain, who had also received a previous diagnosis of MCS. We studied a total of 21 patients; all patients underwent neurological clinical examination (including scales for pain and autonomic disorders) and skin biopsy. Age matched healthy were used as controls for skin biopsy data. In addition, nerve conduction studies and serum screening to exclude possible causes of peripheral neuropathy were also performed. Skin biopsy disclosed a somatic SFN in all patients. Notwithstanding the majority (18 out of 21) of patients complained also of autonomic symptoms we found a sparing of autonomic innervation on skin biopsy. Chronic pain in MCS could be secondary to the presence of a somatic SFN although larger studies are needed to confirm our observation.

\section{Introduction}

Small fiber neuropathy (SFN) is an often misdiagnosed condition characterised by either sensory and/or autonomic symptoms due to a selective involvement of $A \delta$ and C-fibres. Diagnosis is based mainly on skin biopsy, quantitative sensory testing and clinical questionnaires although other examination such as test of sudomotor function can also play a role. [1] Multiple chemical sensitivity (MCS) syndrome is a still not fully understood condition where patients reports the arising of different symptoms after exposure to several chemical substances. [2] Among the symptoms reported, pain is often present in patients who complain of MCS symptoms. [2] In this paper we report the results of a study conducted in a cohort of patients referred to our observation for the suspect of SFN because of chronic pain who had received a previous diagnosis of MCS syndrome.

\section{Materials And Methods}

We screening 25 patients referred to our observation because of chronic pain and a previous diagnosis of MCS from Italian tertiary centers particularly skilled in the diagnosis of MCS. However, 4 patients were excluded from the study because the presence of acquired causes predisposing a peripheral nerve involvement such as Sjogreen disease (2 patients) and vitamin B12 deficiency (2 different patients). For this study we selected a total of 21 patients ( $19 \mathrm{~F}, 2 \mathrm{M}$, mean age 50.8 years +-8.53) showing no predisposing causes for peripheral neuropathy after an extensive serum screening excluding diabetes, liver, kidney and thyroid dysfunctions, vitamin B12 and folate deficiencies, autoimmune disorders and infections. The MCS diagnosis was performed based on previous described criteria. [2] Diagnosis of the condition was based on the presence of at least 6 months of recurrent symptoms involving central nervous system (CNS) associated with self reported smell hypersensitivity and at least another organ system: the main feature of the condition is that symptoms are triggered by exposure to low levels of 
multiple chemicals substances and ameliorates or disappear after removal of the noxious stimuli. [2] Besides CNS involvement patients often complains of allergic diathesis, self-reported food or alcohol intolerance and symptoms regarding musculoskeletal, gastrointestinal, skin, respiratory, peripheral nerves and cardiovascular system. [2,3]. In addition, 16 out of 21 patients also received the diagnosis of fibromyalgia. All patients underwent neurological clinical examination (including clinical scales for pain and autonomic disorders), nerve conduction studies and skin biopsy. Skin biopsy results were compared to age matched healthy controls ( $15 \mathrm{~F}, 4 \mathrm{M}$, mean age 57.22 years +- 13.081). The procedures used were approved by the local Human Ethics Committee (Comitato Etico indipendente-AUSL Bologna, code number 12073) and followed the Helsinki Declaration related to clinical research of human beings. In addition, patients and healthy controls gave their written informed consent to participate in the study.

\section{Skin biopsy}

Three $\mathrm{mm}$ punch biopsies were taken from proximal thigh ( $15 \mathrm{~cm}$ above the patella) and distal leg (10 $\mathrm{cm}$ above the lateral malleolus) hairy skin sites. 50-microm-thick sections were obtained using a freezing sliding microtome (HM550; Thermo Scientific, Waltham, MA, USA). Twelve free-floating sections were incubated overnight with a panel of primary antibodies, including mouse (1:750; Abcam, Cambridge, UK; ab72911) or rabbit (1:1000; AbD Serotec, Raleigh, NC, USA; 7863-0504) pan-neuronal marker protein gene product (PGP) 9.5, mouse, collagen IV (CollV; 1:800; Chemicon, Temecula, CA, USA; MAB1910). As autonomic markers, rabbit dopamine-beta-hydroxylase ( $\mathrm{DbH} ; 1: 150$, Chemicon, AB1536) and rabbit vasoactive intestinal peptide, (VIP; 1:1000; ImmunoStar, Hudson, WI, USA; 20,077) were used to identify noradrenergic and sudomotor cholinergic fibers respectively. Sections were then washed and secondary antibodies, labeled with mouse Alexa Fluor 488 and rabbit cyanine dye fluorophores 3.18 (1:400; Jackson ImmunoResearch, West Grove, PA, USA; 715-545-150 for m-Alexa Fluor 488 and 711-165-152 for r-cyanine 3), were added for an overnight incubation. Skin sections were firstly viewed and analyzed under a Zeiss fluorescent microscope. Furthermore, the sections were also analyzed using a confocal laser scanning microscope (Nikon confocal microscopy, Eclipse Ti A1, Japan) to study the innervation pattern. Digital images were collected in successive frames of 1-2 $\mu \mathrm{m}$ increments on a Z-stack plan, at the appropriate wavelengths for secondary antibodies, with a x 20 or x40 plan apochromat objective and subsequently projected to obtain a double-stained digital image with a computerized system. Autonomic innervation density was quantified using the previously described automated method showing high interobserver and intraobserver reliability. [4,5] Epidermal nerve fiber density (ENFD) was calculated by considering a single epidermal fiber marked by PGP 9.5 crossings of the dermal-epidermal junction.

\section{Nerve conduction studies}

Sensory nerve conduction studies were recorded from sural and peroneal nerve bilaterally in the lower limbs, from median or ulnar nerve of the non-dominant hand in the upper limbs. Sensory conduction studies were performed antidromically. Motor fibres conduction studies were performed recording from abductor hallucis brevis and extensor digitorum brevis (tibial and peroneal nerve respectively) bilaterally 
in the lower limbs and from abductor digiti minimi or abductor pollicis brevis in the upper limbs (ulnar or median nerve respectively).

\section{Clinical assessment}

All patients underwent complete neurological assessment and clinical questionnaires assessing neuropathic pain and autonomic symptoms (DN4 and Compass 31 scale, respectively). [6,7]

\section{Statistic}

Statistical analyses were performed using SPSS 25.0 for Windows (IBM Corp., Armonk, NY). Categorical variables were evaluated by chi-square test. For the analysis of continuous variables, we used the Kolmogorov-Smirnov test to verify the normal data distribution. Autonomic innervation density values were normally distributed, and an ANCOVA was used to evaluate differences between patients and healthy controls, using age and sex as covariates. Sensitivity and specificity for differentiating the groups were calculated using the optimal cut-off values determined by receiver operating characteristic (ROC) curve analysis. To search for correlation between skin biopsy data and clinical scores, we used Spearman rank test, as clinical scale values were not normally distributed. P-values $<0.05$ were accepted as statistically significant.

\section{Results}

\section{Clinical assessment and nerve conduction studies}

Patients and controls were matched for sex and age. Neurological examination did not find any abnormal sign in patients although all of them complain of pain mainly at the extremity of the limbs while autonomic symptoms were complained of only by some of them (Table 1). The mean duration of symptoms was 9.6 years for pain (range 1-40; SD 8.78) and 15,38 years (range 2-45; SD 13.73) for autonomic symptoms. DN4 score mean score was 6.5 (range 4-9; SD +-1.43). Mean COMPASS 31 score was 38.5 (range 0-65,7; SD 19.08). For what it concerns the latter, the more involved subdomains resulted the cardiovascular and gastrointestinal ones (adjusted mean score of 16.21 and 9.81 respectively). Scoring of DN4, COMPASS 31 and clinical features of the patients are summarised in Table 2. Nerve conduction studies resulted normal in all the patients.

Table 1. Clinical and demographic features of patients are summarised in the table. Duration of pain and autonomic symptoms is expressed in years at time of evaluation. 21 patients out of 21 complained of pain (mainly localised at the extremities), 18 patients out of 21 complained of autonomic symptoms. 


\begin{tabular}{|c|c|c|c|c|c|c|}
\hline Patient & Sex & Age & PAIN & $\begin{array}{l}\text { Duration of pain } \\
\text { (years) }\end{array}$ & $\begin{array}{l}\text { Autonomic } \\
\text { Symptoms }\end{array}$ & $\begin{array}{l}\text { Duration of autonomic } \\
\text { symptoms (years) }\end{array}$ \\
\hline $\begin{array}{l}\text { Patient } \\
1\end{array}$ & $\mathrm{~F}$ & 46 & Yes & 13 & Yes & 13 \\
\hline $\begin{array}{l}\text { Patient } \\
2\end{array}$ & $\mathrm{~F}$ & 50 & Yes & 7 & NO & // \\
\hline $\begin{array}{l}\text { Patient } \\
3\end{array}$ & $\mathrm{~F}$ & 55 & Yes & 2 & Yes & 2 \\
\hline $\begin{array}{l}\text { Patient } \\
4\end{array}$ & $\mathrm{~F}$ & 51 & Yes & 1 & Yes & 4 \\
\hline $\begin{array}{l}\text { Patient } \\
5\end{array}$ & $F$ & 55 & Yes & 9 & Yes & 15 \\
\hline $\begin{array}{l}\text { Patient } \\
6\end{array}$ & $M$ & 39 & Yes & 7 & NO & // \\
\hline $\begin{array}{l}\text { Patient } \\
7\end{array}$ & $\mathrm{~F}$ & 54 & Yes & 9 & Yes & 20 \\
\hline $\begin{array}{l}\text { Patient } \\
8\end{array}$ & $F$ & 46 & Yes & 9 & Yes & 34 \\
\hline $\begin{array}{l}\text { Patient } \\
9\end{array}$ & $\mathrm{~F}$ & 59 & Yes & 15 & Yes & 15 \\
\hline $\begin{array}{l}\text { Patient } \\
10\end{array}$ & $\mathrm{~F}$ & 48 & Yes & 1,5 & Yes & 3 \\
\hline $\begin{array}{l}\text { Patient } \\
11\end{array}$ & $\mathrm{~F}$ & 76 & Yes & 40 & Yes & 40 \\
\hline $\begin{array}{l}\text { Patient } \\
12\end{array}$ & $F$ & 46 & Yes & 16 & Yes & 30 \\
\hline $\begin{array}{l}\text { Patient } \\
13\end{array}$ & $\mathrm{~F}$ & 41 & Yes & 5 & Yes & 6 \\
\hline $\begin{array}{l}\text { Patient } \\
14\end{array}$ & $F$ & 43 & Yes & 23 & Yes & 23 \\
\hline $\begin{array}{l}\text { Patient } \\
15\end{array}$ & $M$ & 58 & Yes & 10 & Yes & 45 \\
\hline $\begin{array}{l}\text { Patient } \\
16\end{array}$ & $\mathrm{~F}$ & 45 & Yes & 5 & Yes & 5 \\
\hline $\begin{array}{l}\text { Patient } \\
17\end{array}$ & $F$ & 58 & Yes & 4 & Yes & 4 \\
\hline $\begin{array}{l}\text { Patient } \\
18\end{array}$ & $\mathrm{~F}$ & 47 & Yes & 5 & Yes & 5 \\
\hline
\end{tabular}




\begin{tabular}{|lllllll|}
\hline Patient & Sex & Age & PAIN & $\begin{array}{l}\text { Duration of pain } \\
\text { (years) }\end{array}$ & $\begin{array}{l}\text { Autonomic } \\
\text { Symptoms }\end{array}$ & $\begin{array}{l}\text { Duration of autonomic } \\
\text { symptoms (years) }\end{array}$ \\
\hline $\begin{array}{l}\text { Patient } \\
19\end{array}$ & F & 56 & Yes & 3 & Yes & 3 \\
\hline $\begin{array}{l}\text { Patient } \\
20\end{array}$ & F & 55 & Yes & 10 & Yes & 10 \\
\hline $\begin{array}{l}\text { Patient } \\
21\end{array}$ & F & 39 & Yes & 7 & NO & $/ /$ \\
\hline
\end{tabular}

Table 2. Clinical features of the patients and results on pain and autonomic symptoms scales are summarised in the table. The mean duration of symptoms was 9.6 years for pain (range 1-40; SD 8.78) and 15.38 years (range 2-45; SD 13.73) for autonomic symptoms. DN 4 score mean score was 6.5 (range 4-9; SD +-1.43). Mean COMPASS 31 score was 38.5 (range 0-65.7; SD 19.08). For what it concerns the latter, the more involved subdomains resulted the cardiovascular and gastrointestinal ones (adjusted mean score of 16.21 and 9.81 respectively). 


\section{Clinical features}

Number of patients complaining of pain

Mean duration of pain at time of evaluation

DN4

Number of patients complaining of autonomic symptoms

Mean duration of autonomic symptoms at time of evaluation

Compass 31 Total score

Compass 31 Cardiovascular Domain

Compass 31

Skin Domain

Compass 31

6.42 (range 0-12.85; SD 3.70)

Salivary and Lacrimal Domain

Compass 31

9.81 (range 0-17.85; SD 5.29)

Gastrointestinal Domain

Compass 31

2.09 (range 0-14.28; SD 3.23)

Urinary Domain

Compass 31

2.13 (range 0-3.99; SD 1.54)

\section{Intrinsic eye motility Domain}

\section{Skin innervation}

ENFD was significantly decreased in both thigh and leg while the autonomic innervation did not differ from controls (Table 3; Figure 1). The appropriate cutoff ENFD score differentiating patients from controls was calculated by using the ROC curve analysis. An optimal cutoff score in the lower leg was 8.3 ENFD/mm (sensitivity $93 \%$ and specificity $100 \%$ ) and $12.8 \mathrm{ENFD} / \mathrm{mm}$ (sensitivity $86 \%$ and specificity $100 \%)$ for the thigh. Adopting these scores a somatic small fiber neuropathy was found in all patients: in 18 patients ( $86 \%$ ) with a distal and proximal involvements, 2 patients $(10 \%)$ showed only a proximal neuropathy while 1 patient (4\%) presented only a distal involvement (Figure 2).

The correlation analysis disclosed no significant correlation correlation between ENFD (in both leg and thigh) and the degree of neuropathic pain evaluated by DN4 $(p>0,05)$ or the pain duration $(p>0,05)$ although a slight tendency toward an inverse correlation was found between thigh ENFD and DN4 score $(r=-0,4 ; p=0,07)$. Moreover, no correlation between autonomic skin innervation and COMPASS 31 score, including the correlated subdomains, was found. 
Table 3. Skin biopsy somatic and autonomic innervations mean results of the patients and healthy subjects are shown. Epidermal fiber nerve density (EFND) is calculated counting every single epidermal fiber marked with PGP 9.5 that crosses the dermal-epidermal junction. EFND is calculated for every sample, the site (thigh or leg) EFND is then calculated as a mean of the three samples obtained from each site. Arrector pili muscle (APM) innervation was calculated counting the number of fibres marked with Dopamine beta hydroxylase (noradrenergic marker) surrounding these structures of the hair follicles. Sweat glands (SG) innervation was calculated considering the number of fibres marked with vasoactive intestinal peptide (cholinergic marker). We found no differences between patients and healthy control in autonomic innervation parameters.

\begin{tabular}{|lll|}
\hline & Patients & Control \\
\hline Thigh (EFND/mm) & $9.91(+-2.59)$ & $19.9(+-4.6)$ \\
\hline Leg (EFND/mm) & $6.06(+-2.22)$ & $13.5(+-3.1)$ \\
\hline APM thigh (\%) & $19.21(+-2.48)$ & $18.02(+-2.97)$ \\
\hline APM leg (\%) & $15.48(+-3.29)$ & $15.65(+-3.91)$ \\
\hline SG thigh (\%) & $15.73(+-1.72)$ & $14.88(+-1.98)$ \\
\hline SG Leg (\%) & $15.13(+-2.24)$ & $13.71(+-1.86)$ \\
\hline
\end{tabular}

\section{Discussion}

Multiple chemical sensitivity is a still not full known disorder which unfortunately to date lack of objective identifiable alteration. The clinical picture is characterized by multitude of symptoms which are triggered by exposure to low concentrations of various chemical substances. The condition is also known as Idiopathic environmental intolerance. $[2,8,9]$ However, the triggering substances and the concentration level required to provoke symptoms are not well defined and there is an huge variability among patients. MCS is not rare and epidemiological studies suggest it can affect up to $13 \%$ of population. $[10,11]$ The pathogenic mechanism is still not understood; a possibile role of inflammatory cytokines, immune cell abnormalities, metabolic vulnerability to oxidative stress and neural sensitization have been suggested. [12-17] Genetic polymorphisms of the superoxide dismutase (SOD) 2 gene has also been proposed to increase the risk of being affected by the condition. [18] Moreover, polymorphisms involving the gene encoding for CYP2D6 and NAT-2 (two enzymes involved in drugs metabolism) have also being reported as factors which increase the likelihood of being affected by MCS. [19] Among the most common symptoms reported by patients there are ortosthatic intolerance, subjective breathing difficulties, rash and peripheral edema. [2, 3, 20] Psychiatric comorbodities are also very common. [21] In addition, patients with MCS very often complained of chronic pain which is a disabling symptom with a severe impact in quality of life. $[2,3,20]$ Chronic pain is also very common in a fibromyalgia disorder and chronic fatigue 
syndrome (CFS). [22] Fibromyalgia is a frequent disorder with an estimated prevalence of about $3-5 \%$ in normal population. $[23,24]$ The condition is characterized by widespread pain and tenderness in specific parts of the body (the so called "tender points"). The mechanism of pain in fibromyalgia is mainly considered related to a central abnormal amplification of pain. However up to $60 \%$ of patients diagnosed with fibromyalgia have a reduced intraepidermal nerve fiber density. [25-29] Moreover microneurographic evaluations of patients affected by fibromyalgia showed also an abnormal activities in C fibres. [30] The main clinical feature of CFS is a disabling fatigue lasting at least 6 month which is neither provoked by exercise or relieved by rest, is present for the most part of the day and is associated with other symptoms including pain, concentration difficulties and sleep disturbance. [31] Our data showed the presence of a somatic small fiber neuropathy in all examined MCS patients complaining pain. This is the first study in literature reporting the presence of SFN in patients affected by chronic pain and MCS. Although the lack of correlation between DN4 and ENFD could suggests that chronic pain in MCS patients is not related to the presence of an underlying small fiber neuropathy, this result is not surprising as it has previously been described that neuropathic pain is not correlated to the epidermal innervation. [32]

This finding could be due to the universally use of a pan-neural (i.e. PGP 9.5) not reflecting the complexity of nociceptor subgroups (i.e. damaged and regenerating fibers) involved in SFN. [32]

Furthermore, SFN has been described in conditions classically characterized by absence of pain as amyotrophic lateral sclerosis [33].

Notwithstanding the foregoing, the slight tendency towards an inverse correlation between thigh ENFD and DN4 score support a possible influence of epidermal denervation in the pain symptoms complained by our MCS patients.

Moreover it should be mentioned that the majority of our patients had received also a previous diagnosis fibromyalgia; as the presence of SFN can be found up to $60 \%$ of patients affected by fibromyalgia but all of our patients showed a reduced EFND, an additional different mechanism behind the overlap underlying the pain in the two conditions is likely. Interestingly we found a sparing of the autonomic skin innervation component suggesting that autonomic symptoms complained by patients and assessed through COMPASS 31 scale are possibly due to a selective involvement of specific autonomic subdivisions (such as the cardiovascular and gastrointestinal systems) sparing the skin or could have a different underlying pathogenic process.

We were unable to perform cardiovascular reflexes to ascertain a cardiovascular autonomic involvement in our patients and this configures a limitation of the study. The main reason that prevented us from performing cardiovascular reflexes was the inability of the patients to stay in the hospital for a long time.

Notwithstanding the suggestive possibility of having found a pathological biomarker for pain complained by MCS patients, our data do not automatically imply that SFN is part of the MCS clinical picture; as we study a subset of patients who were referred to our observation for the presence of chronic pain an 
additional study involving a larger cohort of MCS patients, hopefully involving subjects without pain, is needed to establish the relevance of SFN in these patients.

The study of patients without pain could be interesting since SFN has been reported as an early pathological sign of other disease, such as diabetes. $[34,35]$ In accordance with the foregoing, whether SFN is part of the clinical picture of MCS and if so whether it has a causal role in the pain complained by the patients still remained to be better clarified. A functional study of peripheral nociceptors, by using microneurography [36], will be desirable to confirm the nociceptors involvement underlying ENFD abnormalities and pain complained by MCS patients.

\section{Conclusions}

We studied 21 patients with a previous diagnosis of MCS complaining of chronic pain disclosing somatic SFN on skin biopsy in all patients. Notwithstanding high mean score on COMPASS 31 scale we found a sparing of autonomic fibres suggesting a lesser role of skin nerve for autonomic symptoms. This is the first skin biopsy study conducted on MCS patients however to confirm whether somatic SFN is part of the condition future and larger studies should be conducted.

\section{Declarations}

\section{Aknowledgments}

All authors declare no conflicts of interest.

\section{References}

1) Devigili G, Rinaldo S, Lombardi R, Cazzato D, Margherita Marchi M, Salvi E, Eleopra R and Lauria G. Diagnostic criteria for small fibre neuropathy in clinical practice and research.BRAIN 2019: 142; 37283736. doi:10.1093/brain/awz333

2) Lacour M, Zunder T, Schmidtke K, Vaith P, Scheidt C. Multiple chemical sensitivity syndrome: suggestions for an extension of the US. MCS case definition. Int J Hyg Environ Health. 2005;208:141151

3) Black,D.W.,Rathe,A., Goldstein,R.B.,1990. Environmental illness. A controlled study of 26 subjects with '20th century disease'. J. Am. Med. Assoc. 264,3166-3170.

4) Donadio V, Incensi A, Giannoccaro MP, Cortelli P, Di Stasi V, Pizza F, Jaber MA, Baruzzi A, Liguori R. et al (2012) Peripheral autonomic neuropathy: diagnostic contribution of skin biopsy. J Neuropathol Exp Neurol 71:1000-1008 4.

5) Donadio V, Nolano M, Provitera V, Stancanelli A, Lullo F, Liguori R, Santoro et al (2006) Skin sympathetic adrenergic innervation: an immunofluorescence confocal study. Ann Neurol 59:376-381 
6) VanDenKerkhof EG, Stitt L, Clark AJ, Gordon A, Lynch M, Morley-Forster PK, Nathan HJ, Smyth C, Toth C, Ware MA, Moulin DE. Sensitivity of the DN4 in Screening for Neuropathic Pain Syndromes. Clin J Pain. 2018 Jan;34(1):30-36. doi: 10.1097/AJP.0000000000000512. PMID: 28481836.

7) Sletten DM, Suarez GA, Low PA, Mandrekar J, Singer W. COMPASS 31: a refined and abbreviated Composite Autonomic Symptom Score. Mayo Clin Proc. 2012 Dec;87(12):1196-201. doi:

10.1016/j.mayocp.2012.10.013. PMID: 23218087; PMCID: PMC3541923.)

8) MCS consensus conference,1999. Multiple chemical sensitivity: a 1999 consensus. Arch. Environ. Health 54,147-149.

9) Kreutzer,R.,2000. Idiopathic environmental intolerance: case definition issues. Occup. Med. 15,511517.

10) Steinemann A. National Prevalence and Effects of Multiple Chemical Sensitivities. J Occup Environ Med. 2018 Mar;60(3):e152-e156. doi: 10.1097/JOM.0000000000001272. PMID: 29329146; PMCID: PMC5865484.

11) Steinemann A. Prevalence and effects of multiple chemical sensitivities in Australia. Prev Med Rep. 2018 Mar 10;10:191-194. doi: 10.1016/j.pmedr.2018.03.007. PMID: 29868366; PMCID: PMC5984225.

12) Kipen H, Fiedler N, Maccia C, Yurkow E, Todaro J, Laskin D. Immunologic evaluation of chemically sensitive patients. Toxicol Ind Health. 1992 Jul-Aug;8(4):125-35. PMID: 1412479.

13) Bell IR, Schwartz GE, Baldwin CM, Hardin EE, Klimas NG, Kline JP, Patarca R, Song ZY. Individual differences in neural sensitization and the role of context in illness from low-level environmental chemical exposures. Environ Health Perspect. 1997 Mar;105 Suppl 2(Suppl 2):457-66. doi:

10.1289/ehp.97105s2457. PMID: 9167980; PMCID: PMC1469822.

14) De Luca C, Scordo MG, Cesareo E, Pastore S, Mariani S, Maiani G, Stancato A, Loreti B, Valacchi G, Lubrano C, Raskovic D, De Padova L, Genovesi G, Korkina L. Biological Definition of Multiple Chemical Sensitivity from Redox State and Cytokine Profiling and not from Polymorphisms of XenobioticMetabolizing Enzymes. Toxicol. Appl. Pharmacol. 2010;248:285-292.

15) De Luca C, Raskovic D, Pacifico V, Thai JC, Korkina L. The search for reliable biomarkers of disease in multiple chemical sensitivity and other environmental intolerances. Int J Environ Res Public Health. 2011;8(7):2770-2797. doi:10.3390/ijerph8072770

16) Korkina L, Scordo G, Deeva I, Cesareo E, De Luca C. The chemical defensive system in the pathobiology of idiopathic environment-associated diseases. Curr. Drug Metab. 2009;10:914-931.

17) Dearman RJ, Kimber I. Cytokine profiling and chemical allergy. Toxicol. Appl. Pharmacol. 2002;185:228-229. 
18)Cui X, Lu X, Hiura M, Oda M, Miyazaki W, Katoh T. Evaluation of genetic polymorphisms in patients with multiple chemical sensitivity. PLoS One. 2013 Aug 13;8(8):e73708. doi:

10.1371/journal.pone.0073708. PMID: 23967348; PMCID: PMC3742528.

19)McKeown-Eyssen G, Baines C, Cole DE, Riley N, Tyndale RF, Marshall L, Jazmaji V. Case-control study of genotypes in multiple chemical sensitivity: CYP2D6, NAT1, NAT2, PON1, PON2 and MTHFR. Int J Epidemiol. 2004 Oct;33(5):971-8. doi: 10.1093/ije/dyh251. Epub 2004 Jul 15. PMID: 15256524.

20) Black,D.W.,Okiishi,C.,Schlosser,S.,2000a. A nine-year follow-up of people diagnosed with multiple chemical sensitivities. Psychosomatics 41,253-261.

21) Black,D.W.,2000b. The relationship of mental disorders and idiopathic environmental intolerance. Occup. Med. 15, 557-570.

22) Buchwald,D., Garrity,D.,1994. Comparison of patients with chronic fatigue syndrome,fibromyalgia,and multiple chemical sensitivities. Arch. Intern. Med. 154,2049-2053.

23) Clauw DJ, Fibromyalgia A Clinical Review. JAMA April 16, 2014 Volume 311, Number 15

24) Marques AP, et al. Prevalence of fibromyalgia: literature review update. Rev Bras Reumatol Engl Ed. 2017;57(4):356-63

25) Oaklander AL, Herzog ZD, Downs HM, Klein MM. Objective evidence that small-fiber polyneuropathy underlies some illnesses currently labeled as fibromyalgia. Pain. 2013 Nov;154(11):2310-6. doi: 10.1016/j.pain.2013.06.001. Epub 2013 Jun 5. PMID: 23748113; PMCID: PMC3845002.

26) Kosmidis ML, Koutsogeorgopoulo L, Alexopoulos H, Mamali I, Vlachoyiannopoulos PG, Voulgarelis M, et al. Reduction of Intraepidermal Nerve Fiber Density (IENFD) in the biopsies of patients with fibromyalgia: a controlled study. J Neurol Sci 2014;347:143-147

27) Giannoccaro MP, Donadio V, Incensi A, Avoni P, Liguori R. Small nerve fiber involvement in patients referred for fibromyalgia. Muscle Nerve 2014;49:757-759.

28) Uçeyler N, Zeller D, Kahn AK, Kewenig S, Kittel-Schneider S, Schmid A, et al. Small fibre pathology in patients with fibromyalgia syndrome. Brain 2013; 136(Pt 6):1857-1867.

29) Lawson $\mathrm{VH}$, et al. Fibromyalgia syndrome and small fiber, early or mild sensory polyneuropathy. Muscle Nerve. 2018

30) Serra J, Collado A, Sola R, Antonelli F, Torres X, Salgueiro M, et al. Hyperexcitable C nociceptors in fibromyalgia. Ann Neurol 2014;75: 196-208.

31) Cleare AJ, Reid S, Chalder T, Hotopf M, Wessely S (2015). Chronic fatigue syndrome. BMJ Clin Evid 28; pii: 1101. 
32) Truini A, Biasiotta A, Di Stefano G, Leone C, La Cesa S, Galosi E, Piroso S, Pepe A, Giordano C, Cruccu G. Does the epidermal nerve fibre density measured by skin biopsy in patients with peripheral neuropathies correlate with neuropathic pain? Pain. 2014 Apr;155(4):828-832

33) Nolano M, Provitera V, Manganelli F, lodice R, Caporaso G, Stancanelli A, Marinou K, Lanzillo B, Santoro L, Mora G. Non-motor involvement in amyotrophic lateral sclerosis: new insight from nerve and vessel analysis in skin biopsy. Neuropathol Appl Neurobiol. 2017 Feb;43(2):119-132. doi:

10.1111/nan.12332. Epub 2016 Jul 7. PMID: 27288647.

34)Shun CT, Chang YC, Wu HP, Hsieh SC, Lin WM, Lin YH, Tai TY, Hsieh ST. Skin denervation in type 2 diabetes: correlations with diabetic duration and functional impairments. Brain. 2004 Jul;127(Pt 7):1593605. doi: 10.1093/brain/awh180. Epub 2004 May 5. PMID: 15128619.

35)Løseth S, Stålberg E, Jorde R, Mellgren SI. Early diabetic neuropathy: thermal thresholds and intraepidermal nerve fibre density in patients with normal nerve conduction studies. J Neurol. 2008 Aug;255(8):1197-202. doi: 10.1007/s00415-008-0872-0. Epub 2008 Jun 27. PMID: 18574618.

36) Donadio V, Liguori R. Microneurographic recording from unmyelinated nerve fibers in neurological disorders: an update. Clin Neurophysiol. 2015 Mar;126(3):437-45.

\section{Figures}
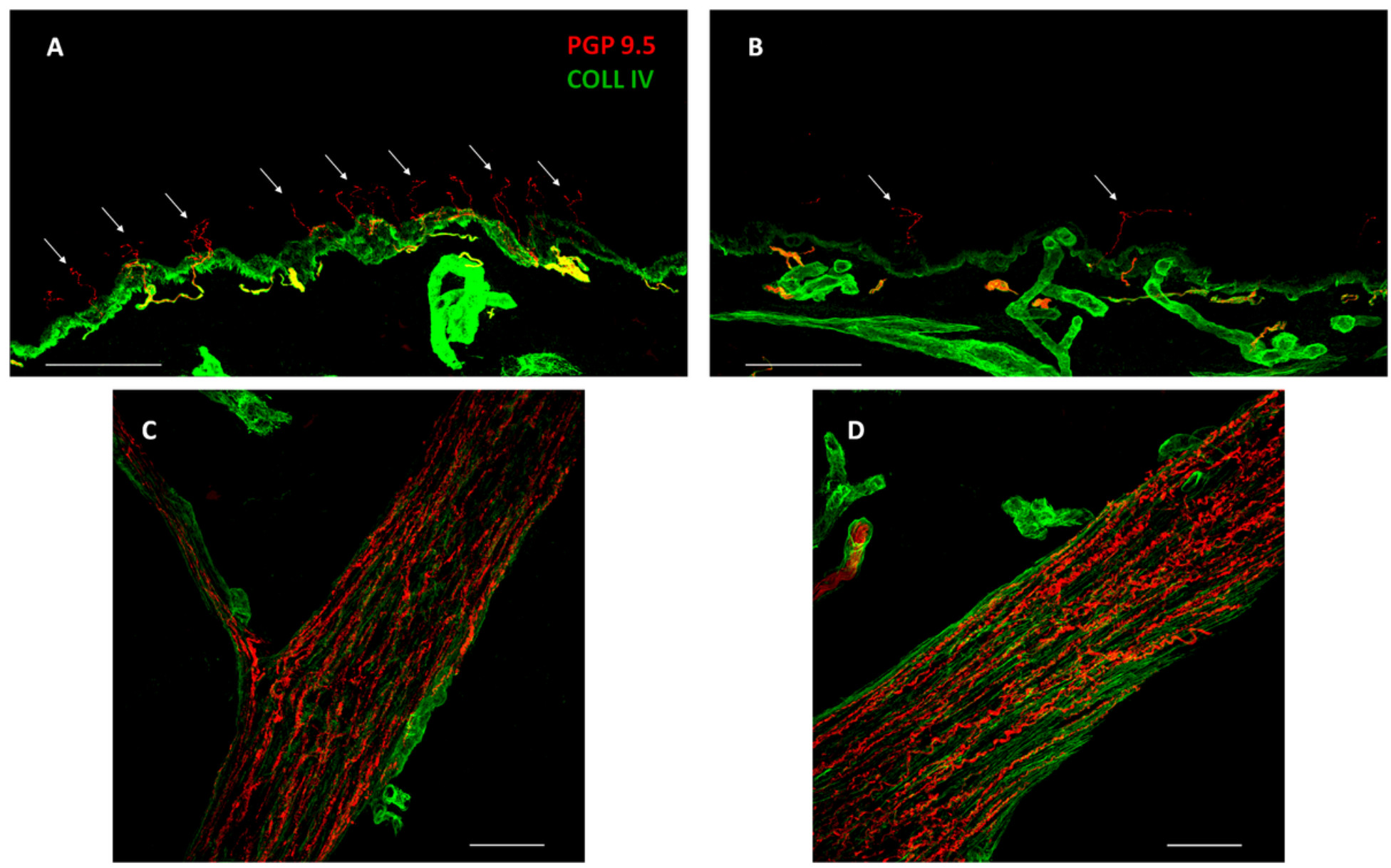


\section{Figure 1}

Confocal study of epidermal and autonomic patterns of innervation in a patient with chronic pain and MCS and a healthy control Figure1. Leg epidermal and autonomic innervation disclosed by confocal microscope ( $\times 20$ in $A$ and $B$ and $\times 40$ in $C$, and $D$ ) in an age-matched healthy subject ( $A$ and $C$ ), and a patient with MCS ( $B$ and $D$ ). Nerve fibers are marked in red using a pan-neuronal marker, PGP 9.5, and collagen staining is shown in green. A-B) Free-ending PGP immunoreactive epidermal fibers (indicated by arrows) are evident in the epidermis of the control (A) whereas MCS patient (B) shows a decrease in the number of such fibers. C-D) Arrector pilum muscle showing a rich density of fibers running in a longitudinal and wavy pattern in both the control (C) and MCS patient (D). Scale bars: $100 \mu \mathrm{m}$ in A and B; $50 \mu \mathrm{m}$ in $\mathrm{C}$ and $\mathrm{D}$ 

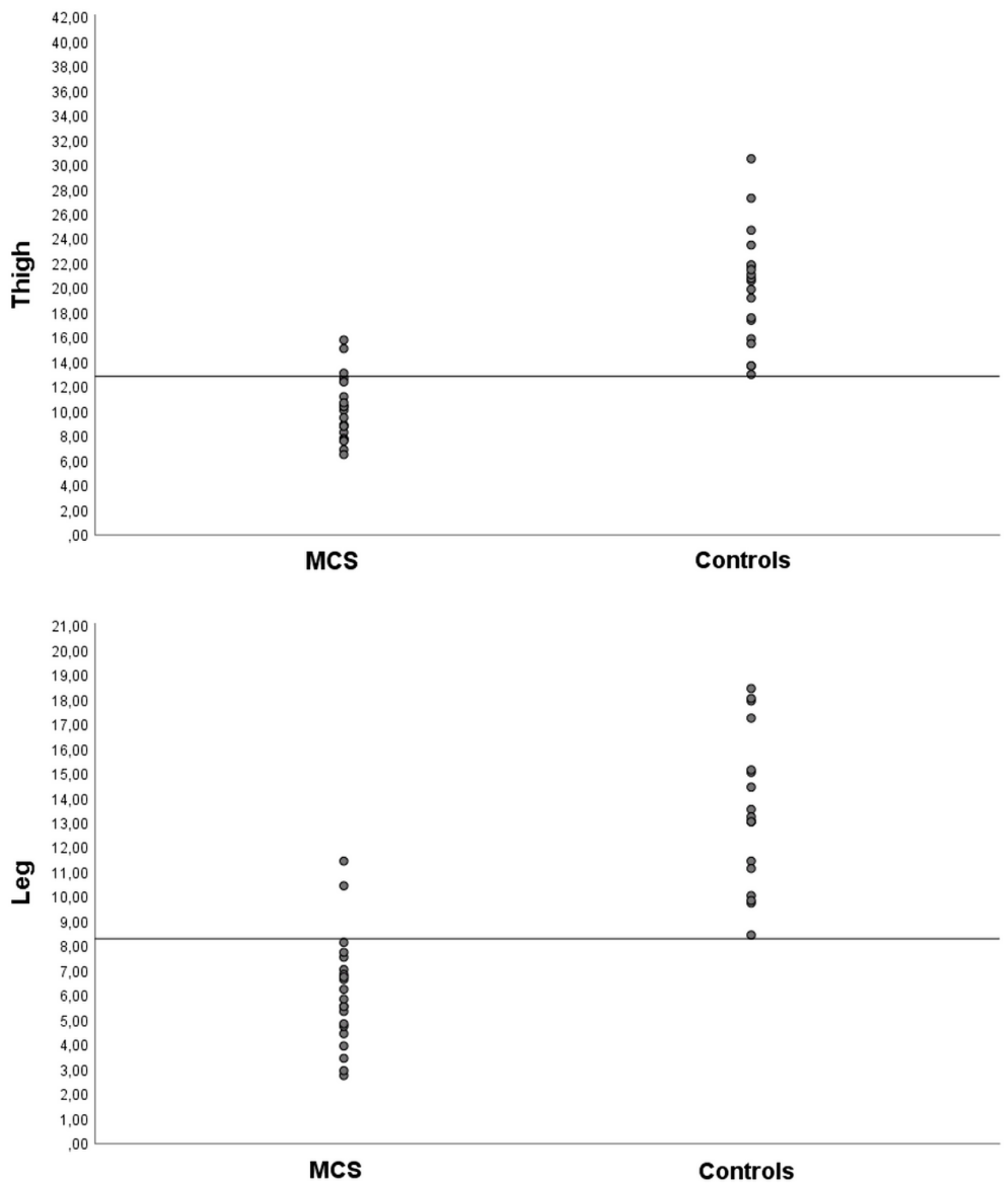

Figure 2

Scatter plot leg and thigh ENFD Figure 2. Scatter plot showing epidermal nerve fiber density (ENFD)/mm in both thigh and leg for patients and con-trols. Patients and controls showed different ENDF/mm values. The optimal cutoff score for differentiating the groups was 8,3 ENFD/mm (sensitivity $93 \%$ and specificity $100 \%$ ) in the lower leg and $12,8 \mathrm{ENFD} / \mathrm{mm}$ (sensitivity $86 \%$ and specificity $100 \%$ ) in the thigh. Adopting these scores a somatic small fiber neuropathy was found in all patients: 17 patients (80\%) disclosed a 
distal and proximal involvements, 2 patients (10\%) showed only a proximal neuropathy while the remaining 2 patient (10\%) disclosed a selective distal involvement. 